To Appear in: 2010 International Symposium on Visual Computing, U.S.A.

\title{
A Novel Consistency Regularizer for Meshless Nonrigid Image Registration
}

\author{
Wei Liu and Eraldo Ribeiro
}

\author{
Computer Vision and Bio-Inspired Computing Laboratory \\ Florida Institute of Technology \\ Melbourne, FL 32901, U.S.A.
}

\begin{abstract}
In nonrigid motion analysis, deformation fields are often modeled using splines defined on a control-point grid. Inspired by recent development of meshfree methods, we propose a novel motion model that does not use control-point grids, nor use explicit node connections. We also propose a regularizer for the deformation field and the minimization algorithm. The method has promising features such as the handling of irregular regions, adaptive accuracy, the multi-scale modeling, and the potential for integrating physical properties into the registration process. Promising results were obtained on both synthetic and real imagery.
\end{abstract}

Keywords: nonrigid meshless image registration, meshfree methods.

\section{Introduction}

Spline-based free-form deformation models have been successfully applied in nonrigid image registration methods $[1,2]$. However, they suffer from a number of problems including the reliance on explicitly connected control points, the conflict between grid resolution and computational efficiency, and the difficulty to handle topological changes. Recent efforts using adaptive irregular grids [2] still rely heavily on explicit control-point connections or meshes, and require nontrivial handling of topology information. In this paper, we propose a novel mesh-free approach for nonrigid image registration that eliminates the need for explicitly handling topology information in the registration process.

Spline-based models' limitation of relying on control-point grids is fundamental, and is shared by applications such as computational mathematics [3], mechanical engineering [4], and computer graphics [5]. Recently, many meshless models (i.e., no explicit control-point connections) have been proposed in different areas $[4,5,3,6,7]$. In this paper, we will focus ourselves on the problem of nonrigid image registration while also drawing from the results in other areas.

For nonrigid image registration, early meshless models are based on radial basis functions (RBFs). For example, the thin-plate spline model [8] represents nonrigid deformations by a linear combination of locally supported RBFs that are scattered over the computation domain. The local basis functions are blended together without explicitly connecting the pieces, and thus avoiding the need for maintaining a control-point grid. Recently, Rohde et al. [8] extended this model 
to adaptively select the number and position of the basis functions according to registration residues. RBFs-based meshless models are also used for data interpolation [3], and for solving partial differential equations (PDEs) [4]. However, RBFs-based models have two main problems. First, as Ruecket et al. [1] pointed out, due to prohibitive computational complexity of the thin-plate spline warps, the registration is restricted to a very limited number of degrees of freedom. Secondly, RBFs are unable to represent polynomial functions exactly (i.e., lack of polynomial reproducibility [4]). For example, RBFs cannot exactly represent a constant deformation $f(\mathbf{x})=c, c \neq 0$. It has been shown that RBFs are less accurate than polynomial-based models in finite-element methods [4]. Here, we propose a polynomial-based meshless model for nonrigid image registration.

Some existing works represent nonrigid deformation using polynomial-based meshless models. For example, polynomial-based moving least-squares (MLS) method has been used for heart-motion analysis [7], and prostate image registration [9]. Most notably, Makram-Ebeid et al. [6] proposed a nonrigid image registration method based on a partition-of-unity finite-element method (PUFE). However, these approaches inherit heavily from meshless methods developed for mechanical engineering, and essentially solve mechanical PDEs using image evidence as boundary conditions. A major difference with respect to previous related work, is that our method does not rely on prior segmentation. Here, we directly integrate the meshless deformation model into the nonrigid image registration formulation, leading to a simplified registration framework.

Our main contributions are as follows. First, we propose a novel image registration method that represents deformation fields by blending together locally supported polynomial models using partition-of-unity (PU) (Section 2). The local deformation models are defined at scattered nodes, and their supporting domains are restricted by radial weighting functions. Secondly, we introduce a new functional to penalize for inconsistencies between local deformation fields (Section 3). This regularizer greatly simplifies the registration process compared to the classic regularizer based on Sobolev norm [1], or to the non-conformity measure proposed in [6]. Additionally, our regularizer is not biased towards certain lower-order deformations. The proposed functional can be minimized hierarchically at varying scales. Unlike previous methods, where a coarse-scale deformation field is only used as an intermediate result [1,6], our method is able to regularize the deformation field by combining image evidence at different scales simultaneously. Finally, these contributions are supported by a number of experiments performed on both synthetic and real images (Section 4).

\section{Meshless deformation model}

We commence by defining the nonrigid image-registration problem as that of deforming a source image $I(\mathbf{x})$ to "best" match a target image $I^{\prime}(\mathbf{x})$ with respect to a given similarity measure [8]. Formally, we seek for a warping field $\mathbf{u}(\mathbf{x})$ that satisfies the following equation $\arg \max _{\mathbf{x}^{\prime}} F\left(I^{\prime}\left(\mathbf{x}^{\prime}\right), I(\mathbf{x}), \mathbf{x}^{\prime}\right), \quad \mathbf{x}^{\prime}=\mathbf{x}+\mathbf{u}(\mathbf{x})$, where $\mathbf{x}$ is a coordinate vector, and $F$ is the intensity-based similarity measure. 


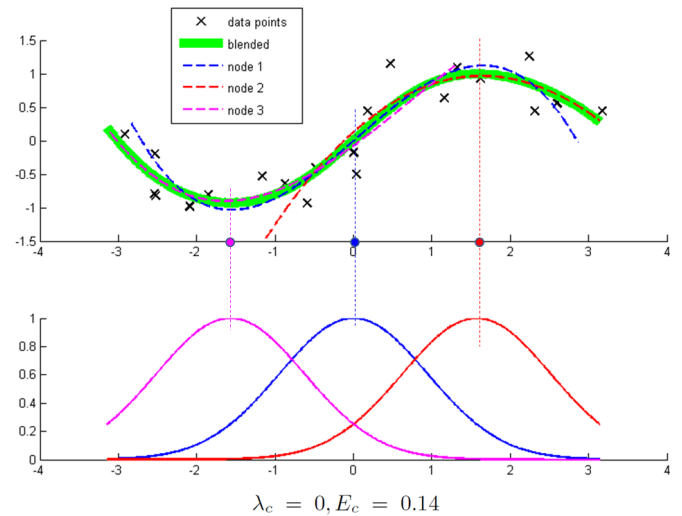

(a)

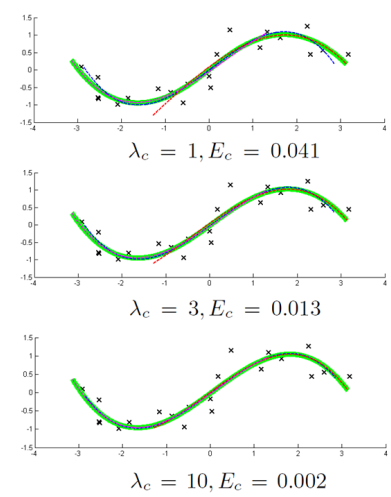

(b)

Fig. 1. Meshless scattered-data approximation using our consistency regularizer. (a) The scattered data-points are locally approximated around each node using polynomials (dashed curves), and then blended into a global approximation (green solid curve) based on their weighting functions (bottom). (b) The consistency regularizer penalizes inconsistencies between local models. Increasing the regularizer coefficient $\lambda_{c}$ leads to more consistent local approximations with lower inconsistency energy $E_{c}$.

Robustness and efficiency are often obtained by representing $\mathbf{x}$ using a sparse set of control points interpolated by splines [1]. To avoid explicit handling of neighborhood information, we approximate the global deformation field $\mathbf{u}(\mathbf{x})$ by blending together a set of particle-based local approximations of the deformation. Thus, no explicit connections between control points are needed. Figure 2 illustrates the concept of our approximation model and consistency regularizer, using an example problem of scattered-data approximation. Here, a set of nodes is distributed across the deformation field domain. Around each node, a local polynomial approximation of the deformation field $\mathbf{u}(\mathbf{x})$ is obtained. As polynomials do not have compact support, we restrict the approximation to the node's vicinity, a region called the node's influence domain. The influence domain will also be used to limit the interaction range between neighboring nodes.

The node's influence domain. Let us define the influence domain $D_{p}$ around a node $p$ as a disk of radius $r_{p}$ (ball in $\left.3-\mathrm{D}^{1}\right) . D_{p}$ can be modeled by a weighting function $w_{p}(\mathbf{x})$ with local support [4]. We define $w_{p}(\mathbf{x})$ as:

$$
w_{p}(\mathbf{x})=\left\{\begin{array}{cl}
\alpha_{p} \exp \left(-\kappa \frac{\|\mathbf{p}-\mathbf{x}\|^{2}}{\sigma_{p}^{2}}\right) & , \mathbf{x} \in D_{p} \\
0 & , \mathbf{x} \notin D_{p}
\end{array},\right.
$$

where $\mathbf{p}$ is the coordinate vector of node $p, \kappa=\frac{1}{9}$, and $\alpha_{p} \in(0,1]$ is the node's predefined influence factor in the final global blending. Thus, a node $p$ is defined

\footnotetext{
${ }^{1} 3$-D extension is straightforward.
} 
by its spatial position, $\mathbf{p}$, the radius (scale) of its influence domain, $\sigma_{p}$, and its influence factor, $\alpha_{p}$. It is worth pointing out that although the weighting function in (1) is a radial function, it is different from previous RBF-based models such as thin-plate splines [8]. Here, the radial basis functions are used for blending the local polynomial models, instead of directly representing the image deformation.

Local approximation model around a node. We write the deformation field $\mathbf{u}_{p}=(u, v)$ around particle $p$ as a linear combination of monomials $\gamma_{j}(\mathbf{x})=x^{s} y^{t}$ :

$$
u(\mathbf{x})=\sum_{j=0}^{m} \gamma_{j}(\mathbf{x}) a_{j} \text { and } v(\mathbf{x})=\sum_{j=0}^{m} \gamma_{j}(\mathbf{x}) b_{j} .
$$

Thus, the deformation field is a linear combination of monomial basis functions $\phi^{\top}(\mathbf{x})=\left[1, x, y, x y, \ldots, x^{s} y^{t}, \ldots\right]$, with coefficients $\mathbf{d}_{p}=\left[a_{0}, b_{0}, \cdots, a_{m}, b_{m}\right]^{\top}$. Monomials in $\phi$ are ordered in a Pascal triangle manner for numerical stability [4]. Next, local deformations $\mathbf{u}_{p}$ are combined into a global deformation field.

Blending local models into a single deformation field. Given the parameters of local deformation models, deformation at point $\mathbf{x}$ can be obtained by blending together the nodes that contain $\mathbf{x}$ in their influence domains. These nodes are called the support domain [4] of $\mathbf{x}$, and are formally denoted by $N_{\mathbf{x}}=\left\{p \| \mathbf{x} \in D_{p}\right\}$. The blended global deformation field is obtained as $\mathbf{U}(\mathbf{x})=$ $\sum_{p \in N_{\mathbf{x}}} r_{p}(\mathbf{x}) \mathbf{u}_{p}(\mathbf{x})$, with $r_{p}(\mathbf{x})=\frac{w_{p}(\mathbf{x})}{\sum_{p^{\prime} \in N_{\mathbf{x}}} w_{p^{\prime}}(\mathbf{x})}$. Here, functions $r_{p}(\mathbf{x})$ ensure the partition-of-unity (PU), i.e., the contributions at $\mathbf{x}$ from various nodes add to one. This blending scheme was used in [6] and is equivalent to the one used in the polyaffine model [10]. The scheme allows for arbitrary placement of local models, while the polynomial basis allows for accurate deformation representation.

\section{Optical flow based registration}

In this section, we integrate the proposed deformation model with squared-sumof-difference (SSD) similarity measure that assumes the image brightness remains constant during image deformation [1]. Study on integrating other similarity measures (e.g., mutual information) is underway. For improved efficiency, the brightness-constancy condition is often linearized to form the optical-flow constraint $I_{x} u+I_{y} v+I_{t}=0[6,2]$. Combining the optical-flow constraint and our meshless deformation model results in an image registration method that is both simple and elegant. It is worth pointing out that the choice of image deformation model is often independent from specific similarity measures, and our meshless deformation model can be easily combined with other similarity measures such as cross-correlation (CC) [8] and mutual-information (MI) [1].

Local intensity constraint. Locally, the weighted sum of squared residues based on optical-flow constraint within a particle's influence domain is given by:

$$
E_{p}^{d}=\sum_{\mathbf{x} \in D_{p}} w_{p}(\mathbf{x})\left(I_{x}(\mathbf{x}) u(\mathbf{x})+I_{y}(\mathbf{x}) v(\mathbf{x})+I_{t}(\mathbf{x})\right)^{2},
$$


where $w_{p}$ is the weighting function defined in Equation 1. By plugging the local model defined in (2) into the registration residual measure in (3), we obtain:

$$
E_{p}^{d}=\left(\mathbf{R}_{p}^{\top} \mathbf{d}_{p}+\mathbf{T}_{p}\right)^{\top} \mathbf{W}_{p}^{d}\left(\mathbf{R}_{p}^{\top} \mathbf{d}_{p}+\mathbf{T}_{p}\right),
$$

where $\mathbf{W}_{p}^{d}$ is the weighting function written as a diagonal matrix, the columns of $\mathbf{R}_{p}$ are the vectors $\left[I_{x}(\mathbf{x}) \gamma_{0}(\mathbf{x}), I_{y}(\mathbf{x}) \gamma_{0}(\mathbf{x}), \ldots, I_{x}(\mathbf{x}) \gamma_{s}(\mathbf{x}), I_{y}(\mathbf{x}) \gamma_{s}(\mathbf{x}), \ldots\right]_{p}^{\top}$ for $\mathbf{x} \in D_{p}, \mathbf{T}_{p}$ is the vector form of $I_{t}(\mathbf{x})$, and $\mathbf{d}_{p}=\left[a_{0}, b_{0}, \ldots, a_{m}, b_{m}\right]_{p}^{\top}$ is the coefficient vector. Equation 4 summarizes the local image evidence.

Consistency enforcement. In spline-based methods, the global deformation field is consistent across control points, and regularization is obtained by penalizing the deformation's spatial variation. In our framework, global deformation fluctuations cause inconsistencies among local deformation fields (Figure 2). As a result, rather than penalizing deformation's spatial variation $[6,2,1]$, we penalize the variation's spatial inconsistency. Indeed, this produces a regularizer that is not biased towards the deformation field's lower-order fluctuations, provided the fluctuation itself is spatially consistent. Consistency between two local deformation fields, $\mathbf{u}_{p}$ and $\mathbf{u}_{q}$, can be measured from the coefficient vectors $\mathbf{d}_{p}$ and $\mathbf{d}_{q}$. However, $\mathbf{u}_{p}$ and $\mathbf{u}_{q}$ lie on different local coordinate systems, and therefore need to be aligned (shifted). Shifting the basis functions $\phi$ by $\boldsymbol{\Delta} \mathbf{x}=[\delta x, \delta y]$ leads to:

$$
\begin{aligned}
\phi(\mathbf{x}+\boldsymbol{\Delta} \mathbf{x}) & =\left[1, x+\delta x, y+\delta y,(x+\delta x)(y+\delta y), \ldots,(y+\delta y)^{m}\right]^{\top} \\
& =\mathbf{S}^{\top}(\boldsymbol{\Delta} \mathbf{x}) \phi(\mathbf{x}),
\end{aligned}
$$

where $\mathbf{S}^{\top}(\boldsymbol{\Delta} \mathbf{x})$ is a linear basis-shifting-operator that can be written as a matrix. Therefore, shifting the local coordinate system leads to shifted coefficients. The local deformation consistency between two nodes $p$ and $q$ can be defined as:

$$
E_{p, q}^{c}=\left[\mathbf{S}^{\prime}(\mathbf{p}-\mathbf{q}) \mathbf{d}_{q}-\mathbf{d}_{p}\right]^{\top}\left[\mathbf{S}^{\prime}(\mathbf{p}-\mathbf{q}) \mathbf{d}_{q}-\mathbf{d}_{p}\right] .
$$

Here, the operator $\mathbf{S}^{\prime}(\mathbf{p}-\mathbf{q})$ is obtained by simply duplicating and shifting the elements of the basis-shifting-operator $\mathbf{S}(\mathbf{p}-\mathbf{q})$ so that the multiplication holds. We can now combine the local data term and the global consistency term into a single functional-minimization problem as follows:

$$
\mathbf{d}_{p}=\underset{\mathbf{d}_{p}}{\arg \min } \sum_{p}\left[E_{p}^{d}+\lambda_{c} \sum_{q \in N_{p}} w_{q}(\|\mathbf{p}-\mathbf{q}\|) E_{p, q}^{c}\right] .
$$

Here, $\lambda_{c}$ defines the consistency regularizer's relative importance. Instead of using a single parameter to control the trade-off between data terms and regularizers $[6,1]$, Equation 7 allows for different nodes to influence the smoothness regularizer, which suggests a potential regularizer adapted to image content. We implemented a Levenberg-Marquardt algorithm to solve the minimization. 


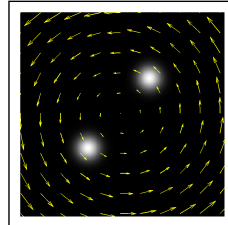

(a)

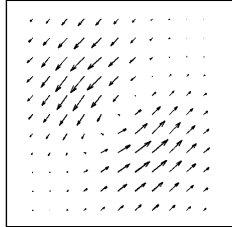

(b)

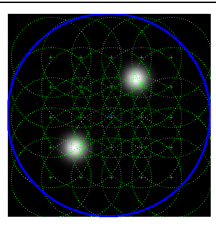

(c)

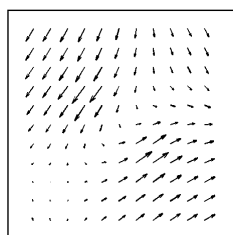

(d)

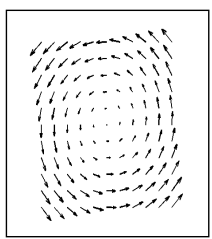

(e)

Fig. 2. Nodes' coupling with varying scales. (a) Ground truth. (b) B-spline registration (Ruecket's [1]). (c) A global node (in blue) with regularly distributed smaller nodes (in green). (d) Our method without global node. (c) Our method with the global node.

Hierarchical warping and multi-scale coupling. We adopt a hierarchical warping process to avoid local minima and improve computational efficiency. Convergence is achieved using a coarser-to-fine manner. Rather than downsizing the image [6], we simply use large scale nodes for coarse registration. In this case, less nodes are needed at large scales, leading to computation efficiency without sacrificing image data. Our framework allows for randomly distributed nodes of different scales. More interestingly, both coarse and fine deformation estimations can be considered simultaneously. This multi-scale ability leads to interesting results as shown in Figure 2. The synthetic example shows a counter-clockwise rotation about the image center with little data evidence. In this case, many ambiguous solutions exist. For the demons [13] and spline-based [1] methods presented in this example, the lack of higher-level information causes a dominant smoothness term to exaggerate deformations at textureless regions. By integrating a node at global scale (blue circle in Figure 2(c)), the simpler rotation solution becomes favored by our energy functional, even though that node's influence power is very small (less than $1 \%$ of the smaller node's weight). While both the exaggerated and the rotation solutions are legitimate, the rotation field is simpler and arguably more consistent with human perception. This large-scale node incorporates into the minimization higher-level information about the deformation field's shape, with little effect on smaller-scale image evidence.

\section{Experiments}

Experiments were performed on two data sets. We first used synthetic fluid PIV images obtained from the FLUID Project [11] which contains seven sequences varying from simple vortices to flows around obstacles. The database's low-speed fluids suits our evaluation, and registration algorithms produce comparable results to fluid-motion estimation methods. Secondly, we used 2-D cardiac MRI data from [12], and compared our method with Rueckert's B-spline based method [1], and an improved Demons method implemented by [13]. 


\subsection{Parameter settings}

Similarly to both finite-element and meshless methods [4], the accuracy of our representation increases with the density of nodes (the $h$ problem), and on the order of basis polynomials (the $p$ problem) for a higher computational cost. We experimentally found that second-order polynomials $\left\{1, x, y, x y, x^{2}, y^{2}\right\}$ provide sufficient representation accuracy at reasonable computational cost. In all experiments, we used second-order polynomials as basis functions. Second-order polynomials are also the empirical choices in many meshless algorithms $[4,6]$. Both the PIV and MRI images are of the size $256 \times 256$ pixels, and we used a grid of $21 \times 21=441$ nodes, i.e., the inter-node spacing was roughly $d=12$ pixels. Finally, in meshless methods, the nodes' average radius $r_{a}$ controls the average number of interacting neighbors (i.e., bandwidth of the system). Higher bandwidth often increases accuracy and stability at the cost of computation. The average radius $r_{a}$ can be defined relative to the inter-node spacing $d$, i.e., $r_{a}=k \times d$, for some $k>0$. We experimentally chose $k=2.5$, so that the interacting neighbors average number was around 20 .

For the consistency regularizer, we chose unity influence factor for all nodes, i.e., $\alpha_{p}=1$, and then roughly tuned the weighting parameter $\lambda_{c}$ on PIV sequences. Experiments show that $\lambda_{c}=10^{-2}$ produced the best registration results. For Rueckert's method, we chose the same control-point density as our meshless method, i.e., a grid of $21 \times 21=441$ control-points. Again, regularizer parameter $\lambda$ was tuned to the PIV sequences, and set $\lambda=10^{-3}$. For the Demon method, we chose the default parameters provided by [13].

\subsection{Analytic fluids}

Regularly and randomly distributed nodes. First, a regular node distribution was used. Secondly, nodes' positions were randomly perturbed (standard deviation of $[-8,8]$ ), while nodes' radii were randomly generated, $r_{p} \in[32,48]$. Finally, for all test cases, we added 15 percent of independent random noise to the input images. Tables 1 and 2 show the results for the different registration methods. Here, we borrowed the Average End-Point Error and the Average Angular Error metrics from the optical-flow literature [2]. The meshless method with regularly distributed nodes produced the best registration results under both metrics. When using randomly perturbed nodes, the nodes' density and registration accuracy are downgraded in some regions, but our method still produced better results, with good robustness to image noise.

Handling topology challenges. In Figure 3, we show a FLUID sequence (Sequence 6) containing a flow around a cylindric obstacle. When using our method with regularly-distributed nodes or a control-point grid for the splinebased method, the obstacle region was estimated as part of the flow (Figure 3(c)). In our method, we can handle holes in the blended global deformation by simply removing the nodes from the regions containing the obstacle (Figure $3(\mathrm{~d})$ ). 
Table 1. Average End-Point Error on Analytic Fluid Sequence

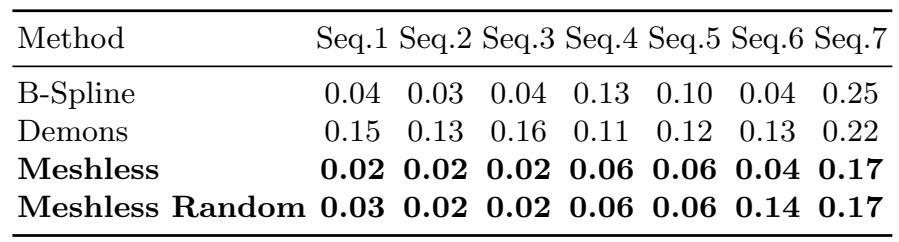

Table 2. Average Angular Error on Analytic Fluid Sequence

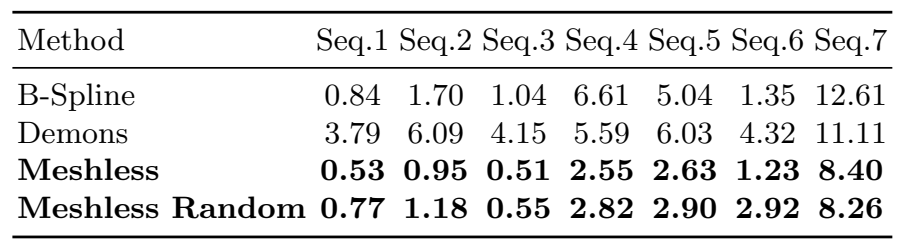

\subsection{Cardiac MRI sequence}

Here, we used cardiac MRI slices from a dataset containing of 33 4-D MRI sequences from different patients [12]. Sequences have 20 frames of a $3-\mathrm{D}$ cardiac motion. We performed intra-subject registration of 2-D slices by registering each slice to the next frame in the sequence. As ground truth was not available in this case, we used both Root-Mean Square (RMS) and Cross-Correlation (CC) as error metrics. While RMS and CC only provide visual verification of the registration results, they still provide good indication of registration quality. Evaluation of our meshless method using clinical images with manually labeled ground-truth data is currently underway and will be reported in due time. Two experiments on the MRI data were performed. First, we used regularly-distributed nodes, and then, node distribution was manually adapted to an area of interest.

Regularly distributed nodes. Parameters for the compared algorithms were set to the same values used for the analytic flows experiment. Table 4.3(right half) shows the error metrics for the methods. Ours underperformed compared methods, but our results were close to Rueckert's method. Next, we describe how results were improved by concentrating nodes around the heart's region.

Adapted registration. In Figure 4(a), we show a polygonal mask manually created on the first frame of a heart sequence. This mask labels the area of interest. Also, the deformation field in the unmasked region was not used for error evaluation. The initial mask was propagated to the remaining frames using the estimated deformation field. As the sequence is relatively short, the mask was reliably kept around the heart. For both Rueckert's and Demons methods, the evaluation was also restricted to the masked area. For our meshless method, the registration process was adapted by removing the nodes outside the mask. Removing nodes helped reduce computation costs significantly allowing for increased node density, by reducing both the space between them and 


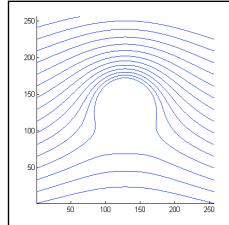

(a)

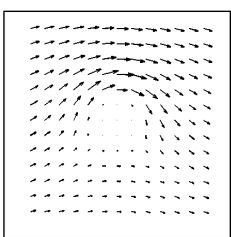

(b)

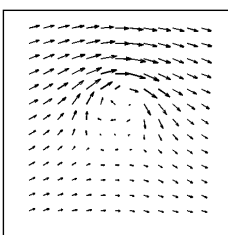

(c)

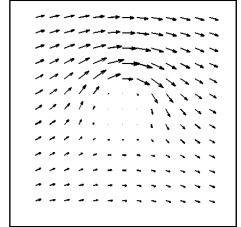

(d)

Fig. 3. Fluid around an obstacle. Grid-based methods overestimate the flow. Our method produces accurate results by removing nodes from the obstacle region. (a) streamline, (b) ground truth, (c) regular, and (d) adapted.

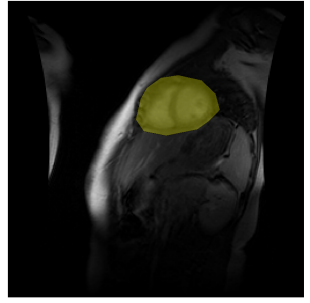

(a) Initial mask

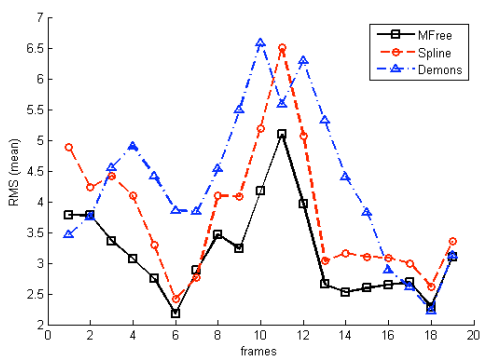

(b) Error in a sequence

Fig. 4. Adapted computation for cardiac motion. (a) Manual initialization of a computation mask. (b) Registration RMS in the sequence for a patient.

their scales by half. Furthermore, rather than initializing a regular control-points grid for each frame [1], we simply propagated the nodes to the next frame. Table 4.3(left half) shows a comparison of the three methods. Here, our method outperformed the other two on almost all metrics. We also observed that after adapting the evaluation, Rueckert's method scored slightly higher than Demons. In Figure 4(b), we show the RMS for registration performed by the three methods on the sequence for a patient. The RMS peak was due to the heart's contraction.

\section{Conclusions}

We proposed a meshless method for nonrigid image registration in which the deformation field is locally modeled using monomials, and then a global deformation field is obtained by blending together the local deformation fields. For regularizing the deformation, we designed an energy function that penalizes inconsistencies between local fields. Quantitative and qualitative evaluations were performed on analytic fluid images, and on cardiac MRI images. Future work includes a comprehensive evaluation of the parameters' effects on registration results, and an extensive comparison to other registration methods. 
Table 3. Node distribution scheme

\begin{tabular}{lcccccccc}
\hline & \multicolumn{3}{c}{ Regularly distributed } & & & \multicolumn{3}{c}{ Adaptively distributed } \\
\cline { 2 - 3 } \cline { 8 - 9 } Method & RMS & RMS & CC & & RMS & RMS & CC \\
mean & variance & mean & & mean & variance & mean \\
\hline B-Spline & 1.45 & 1.08 & 99.75 & & 3.72 & 4.08 & 99.75 \\
Demons & 1.27 & 0.83 & 99.80 & & 4.24 & 3.42 & 93.95 \\
Meshless & $\mathbf{1 . 4 9}$ & $\mathbf{1 . 0 7}$ & $\mathbf{9 9 . 7 3}$ & & $\mathbf{3 . 0 2}$ & $\mathbf{2 . 8 3}$ & $\mathbf{9 8 . 1 5}$ \\
\hline
\end{tabular}

\section{References}

1. Rueckert, D., Sonoda, L., Hayes, C., Hill, D., Leach, M., Hawkes, D.: Nonrigid registration using free-form deformations: application to breast MR images. IEEE Trans. on Med. Imag. 18 (1999) 712-721

2. Hansen, M., Larsen, R., Lyngby, D., Glocker, B., Navab, N., Múnchen, G.: Adaptive parametrization of multivariate B-splines for image registration. In: CVPR. (2008) 1-8

3. Buhmann, M.: Radial basis functions: theory and implementations, volume 12 of Cambridge Monographs on Applied and Computational Mathematics (2003)

4. Liu, G.R.: Mesh free methods: moving beyond the finite element method (Second Edition). CRC (2009)

5. Kobbelt, L., Botsch, M.: A survey of point-based techniques in computer graphics. Computers \& Graphics 28 (2004) 801-814

6. Makram-Ebeid, S., Somphone, O.: Non-rigid image registration using a hierarchical partition of unity finite element method. ICCV $\mathbf{5 1 0}$ (2007) 7

7. Wang, X., Metaxas, D., Chen, T., Axel, L.: Meshless deformable models for LV motion analysis. In: CVPR. (2008) 1-8

8. Rohde, G., Aldroubi, A., Dawant, B.: The adaptive bases algorithm for intensitybased nonrigid image registration. IEEE Trans. on Med. Imag. 22 (2003) 1470-1479

9. Chen, T., Kim, S., Goyal, S., Jabbour, S., Zhou, J., Rajagopal, G., Haffty, B., Yue, N.: Object-constrained meshless deformable algorithm for high speed 3D nonrigid registration between CT and CBCT. Medical physics 37 (2010) 197

10. Arsigny, V., Fillard, P., Pennec, X., Ayache, N.: Fast and simple calculus on tensors in the Log-Euclidean framework. In: Medical Image Computing and ComputerAssisted Intervention-MICCAI, Springer (2005) 115-122

11. Carlier, J.: Second set of fluid mechanics image sequences. European Project 'Fluid image analysis and description' (FLUID) http://www.fluid.irisa.fr/ (2005)

12. Andreopoulos, A., Tsotsos, J.K.: Efficient and generalizable statistical models of shape and appearance for analysis of cardiac MRI. Medical Image Analysis 12 (2008) $335-357$

13. Kroon, D.J., Slump, C.H.: MRI modality transformation in demon registration. In: ISBI, Piscataway, NJ, USA, IEEE (2009) 963-966 